\title{
Further evidence on the effects of word abstractness and meaningfulness in paired-associate learning'
}

\author{
A. Dan Yarmey and Allan Paivio \\ UNIVERSITY OF WESTERN ONTARIO
}

\begin{abstract}
Ss learned a paired-associates list in which concrete or abstract nouns, differing in rated imagery, served as stimuli or responses for nonsense syllable associates. Consistent with previous findings involving noun -noun pairs, learning was easier with concrete than with abstract nouns as stimuli, but variation in concreteness-abstractness had no effect on the response side. Furthermore, the facilitating effect of concrete stimuli apparently compensated for any detrimental effect of the less-meaningful syllable responses. The data provided additional support for a theory in which stimulus items are assumed to function as "conceptual pegs" for their associates.
\end{abstract}

\section{Problem}

Prior related studies (Paivio, 1965; Paivio \& Olver, 1964; Paivio, Yuille, \& Smythe, submitted ${ }^{2}$ ) have demonstrated that a facilitating effect of noun concreteness in paired-associate (PA) learning is greater on the stimulus than on the response side of pairs. Furthermore, rating data indicated that concrete nouns exceed abstract nouns in the ease with which they elicit images, and PA recall scores correlated more highly with stimulus than with response imagery. These findings were predicted from a metaphorical hypothesis (Paivio, 1963) in which stimulus items are assumed to function as "conceptual pegs" for their associates, the efficiency of words as pegs depending on their capacity to evoke images (conditioned sensations) which could mediate response recall. Evidence was also found (Paivio \& Olver, 1964; Paivio et al, submitted) that the stimulus effectiveness of imagery is independent of a correlated attribute, associative meaningfulness (m), which consistently has its greater effect on the response side (e.g., see Underwood \& Schulz, 1960).

To date, the experiments on the effect of concreteness in PA learning have involved a procedure of alternate learning and recall trials using mixed lists of nounnoun pairs, with number of correct recalls as the dependent variable. In addition, with one exception (Paivio \& Olver, 1964), the pairs have been presented auditorily to $\mathrm{Ss}$ in groups. The present investigation sought to confirm the stimulus effectiveness of concreteness and imagery using the more conventional anticipation method of presentation, with independent rather than mixed lists in which either concrete or abstract nouns (differing in imagery but equated on $\mathrm{m}$ ) served as stimuli or responses for nonsense syllable associates. It was ex- pected that learning would be faster with concrete than with abstract nouns as stimuli, but that variation in abstractness would have less effect on the response side. Furthermore, inasmuch as it can be assumed that familiar nouns exceed nonsense syllables in $\mathrm{m}$, comparisons of the effects of concreteness and $\mathrm{m}$ were possible as well. The usual effect of response $m$ would be reflected in easier learning of syllable-noun than of nounsyllable pairs. It was predicted, however, that such an effect would be modified by stimulus concreteness, so that the syllable-noun pairs would be learned more easily than the reversed pairs when the nouns are abstract but not when they are concrete.

\section{Method}

The Ss were 80 Introductory Psychology course students. Twenty (10 of each sex) were randomly assigned to each of four PA lists, which consisted of eight nonsense syllable-noun pairs. The syllables served as stimulus terms for two groups of $\mathrm{Ss}$, the noun associates being concrete for one of these groups and abstract for the other. For the other two groups the pairs were "turned over," so that the nouns served as stimuli. The syllables, of $70-80 \%$ association value (Kreuger, in Underwood \& Schulz, 1960), were the same in each list, viz., bap, nud, pim, ceb, fyd, rez, gac, and nof. The nouns were selected from ones on which $\mathrm{m}$ scores and imagery scores (mean ratings of the ease with which nouns elicit sensory images) were available from previous research (Paivio, 1965; Paivio et al, submitted). They were highly familiar, were equated for $\mathrm{m}$, but all the concrete nouns exceeded the abstract in imagery. The concrete nouns were: chair, umbrella, alcohol, monk, wigwam, elephant, dollar, and flower. The abstract nouns were: history, moment, series, fate, virtue, theory, idea, and ability. Standard instructions for the anticipation method of PA learning were employed. The pairs were presented at a 2:2 sec. rate on a memory drum, with a 6-sec. intertrial interval, and were learned to a criterion of two successive errorless trials. (Seven Ss requiring more than 35 trials were discarded and replaced. Two additional Ss were discarded, one because of equipment failure, another because he was not fluent in English.) Four randomizations of the positions of pairs were used to prevent serial learning. Prior to learning the significant units, four practice trials were given to each S using eight adjective-numeral pairs. On all lists, the Ss were required to pronounce the responses. 
Table 1. Mean Number of Trials to Criterion as a Function of Stimulus and Response ConcretenessAbstractness

\begin{tabular}{lcccc} 
& \multicolumn{2}{c}{ Stimulus } & \multicolumn{2}{c}{ Response } \\
& Mean & SD & Mean & SD \\
Concrete & 15.6 & 5.2 & 16.2 & 8.2 \\
Abstract & 22.6 & 7.9 & 15.9 & 6.7
\end{tabular}

\section{Results and Discussion}

The means and standard deviations of the number of trials to criterion for each group are shown in Table 1. An analysis of variance indicated an insignificant effect of stimulus-response position of nouns $(F(1,76)=3.39$; $\mathrm{p}>.05)$, superior learning with concrete than with abstract nouns $(\mathrm{F}(1,76)=4.10 ; \mathrm{p}<.05)$, and, most important for the prediction, a significant interaction of noun position and abstractness $(F(1,76)=5.02 ; p<.05)$. Comparisons of means by $t$ tests indicated, as predicted, that the group for whom concrete nouns served as stimuli required significantly fewer trials to learn the pairs than those for whom abstract nouns were stimuli $(t(38)=3.24 ; p<.01)$, whereas the concrete-abstract difference on the response side is obviously insignificant by inspection. The effects were similar, though smaller, with the mean number of correct anticipations pertrial as the dependent variable: with nouns as stimuli, the concrete and abstract-group means are 5.16 and 4.77, respectively $(t(38)=1.75 ; p<.05,1$-tailed hypothesis); with nouns as responses the means are 5.37 and 5.15 $(\mathrm{t}<1.00)$.

The results may be contrasted with those of Winnick \& Kressell (in press) who varied noun abstractness only on the response side, with nonsense syllables as stimuli, and found superior learning when the nouns were concrete. They also found, however, that the concrete nouns elicited more associates than did abstract nouns (cf. Lambert, 1955; Paivio, 1965), and they suggested, appropriately, that the response effect of abstractnessconcreteness may be due to correlated differences in $\mathrm{m}$. In the present experiment, the concrete and abstract nouns were equated for $\mathrm{m}$ but the former exceeded the latter in their capacity to evoke images. Thus, the superior stimulus effectiveness of the concrete nouns can be attributed to imagery but not to $\mathrm{m}$.

Viewed in another way, the noun position $x$ abstractness interaction also supports the prediction of contrasting effects for stimulus concreteness and response $\mathrm{m}$. Consistent with the usual effect of $\mathrm{m}$, significantly fewer trials were required to learn nonsense syllablenoun pairs than noun-syllable pairs when the nouns are abstract $(t(38)=2.84 ; p<.01)$, whereas the difference is actually in the opposite direction, although insignificant $(t=0.27)$, when nouns are concrete, indicating that the facilitating effect of concrete, high-imagery stimuli entirely compensated for the negative effect of the low-m, syllable responses. Although the precise mechanisms involved require further explication, the results of this experiment are consistent with previous findings (e.g., Paivio, 1965) and support the conceptual peg theory.

\section{Referenees}

LAMBERT, W。E.Associational fluency as a function of stimulus abstractness. Canad. J. Psychol., 1955, 9, 103-106.

PAIVIO, A. Learning of adjective-noun paired-associates as a function of adjective-noun word order and noun abstractness. Canad. J. Psychol., 1963, 17, 370-379.

PAIVIO, A. Abstractness, imagery, and meaningfulness in pairedassociate learning. J. verbal Learn. verbal Behav., 1965, 4, $32-38$.

PAIVIO, A., \& OLVER, M. Denotative-generality, imagery, and meaningfulness in paired-associate learning of nouns. Psychon. Sci., 1964, 1, 183-184.

PAIVIO, A., YUILLE, J., \& SMYTHE, P. Stimulus and response abstractness, imagery, and meaningfulness, and reported mediators in paired-associate learning. (Submitted)

UNDERWOOD, B. J., \& SCHULZ, R. W. Meaningfulness and verbal learning. Chicago: Lippincott, 1960.

WINNICK, W。A。, \& KRESSEL, K. Tachistoscopic recognition thresholds, paired-associate learning, and immediate recall as a function of abstractness-concreteriess and word frequency.J. exp. Psychol., in press.

Noter

1. This research was partially supported by grants to the second author from the National Research Council of Canada (Grant APA-87) and the University of Western Ontario Research Fund.

2. A copy of the paper cited as "submitted" may be obtained by writing to the second author. 